\title{
Aspekte des Fortlebens des Gregor von Nazianz in byzantinischer und postbyzantinischer Zeit
}

\begin{abstract}
ANDREAS RHOBY
Gregor von Nazianz (329/30-390) gehört zu den in Byzanz und darüber hinaus am meisten rezipierten spätantiken Autoren. Mit Fug und Recht kann behauptet werden, dass die theologischen Werke Gregors im theologischen Schrifttum der Byzantiner nach der Bibel die am besten bekannten und am meisten zitierten sind. ${ }^{1}$ Endlos ist nämlich die Anzahl der Zitate und Anspielungen, die auf den Kirchenvater zurückzuführen sind. Gregor ist eine literarische Instanz, die ebenso wie andere spätantike Autoritäten (Basileios der Große, Ioannes Chrysostomos etc.) bei späteren Autoren hohes Ansehen genießt. ${ }^{2}$ Zahlreich sind auch die aus byzantinischer und postbyzantinischer Zeit stammenden Laudatoren, Biographen und Scholiasten dieses Kirchenvaters. ${ }^{3}$ Außerdem lebte Gregor von Nazianz nicht nur im griechischen Schrifttum weiter, sondern wurde auch in anderen Sprachen rezipiert. Sehr bald übertrug Rufinus von Aquileia (ca. 345-411/12) die Predigten des Gregor von Nazianz ins Lateinische. Außerdem wurden Teile seiner Werke auch ins Arabische, Syrische, Armenische, Georgische und Slawische übertragen. Der erste griechische Kommentar zu vier Reden des Gregor von Nazianz ent-
\end{abstract}

1 J. Noret, Grégoire de Nazianze, l'auteur le plus cité, après la Bible, dans la littérature ecclésiastique byzantine, in: J. Mossay (Hrsg.), II. Symposium Nazianzenum. Louvain-la-Neuve, 25-28 août 1981. Actes du colloque international organisé avec le soutien du Fonds National Belge de la Recherche Scientifique et de la Görres-Gesellschaft zur Pflege der Wissenschaft, Paderborn u.a. 1983, 259-266.

2 Ein anonymer volkssprachlicher Threnos, der die Einnahme Athens durch die Türken im Jahre 1456 bedauert, erwähnt unter den hervorragenden Persönlichkeiten der Stadt Gregor von Nazianz, Basileios den Großen und Ioannes Chrysostomos, ed. Th. N. Philadelpheus, Ioto@í $\alpha \tau \tilde{\omega} v$

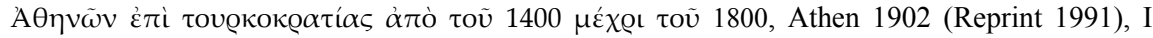
134-136.

3 I. Sajdak, Historia critica scholiastarum et commentatorum Gregorii Nazianzeni. Pars prima (Meletemata Patristica I), Krakau 1914; F. Lefherz, Studien zu Gregor von Nazianz. Mythologie, Überlieferung, Scholiasten, Bonn (Diss.) 1958. Die letzten in Neuedition erschienenen Scholien zu den Reden des Gregor von Nazianz gehören dem Basilius Minimus (10. Jh.) (Basilii Minimi in Gregorii Nazianzeni orationem XXXVIII commentarii ed. Th. S. Schmidt [CC SG 46], Turnhout 2001). 
stammt der Feder des Pseudo-Nonnos und gehört ins 6. Jahrhundert. ${ }^{4}$ In dieser Zeit wird der Nazianzener bereits auch eifrigst zitiert und imitiert: Man denke nur an das von Kaiser Justinian im Jahre 551 erlassene Edictum Rectae Fidei, dessen Prolog über weite Strecken beinahe Wort für Wort nach verschiedenen Diskursen des Kappadokiers zusammengestellt ist. ${ }^{5}$

Die Byzantiner nennen Gregor von Nazianz vor allem ó $\theta \varepsilon \circ \lambda$ ó $\gamma$ os bzw.

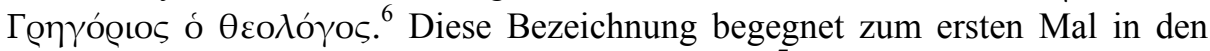
Akten des Konzils von Chalkedon im Jahr 451. ${ }^{7}$ Ein früherer Beleg für ó

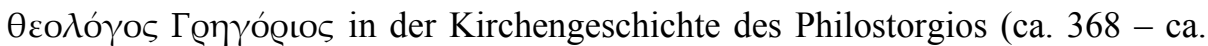
$439)^{8}$ bedeutet nicht, dass dieser Beiname schon damals, also vor Chalkedon, existierte. Die Passage, in der diese Bezeichnung zu finden ist, fußt auf der von Photios angefertigten Epitome, da das Werk des Philostorgios selbst nur in Fragmenten überliefert ist. Mit der Bezeichnung $\theta \varepsilon o \lambda o ́$ o $\varsigma$ tritt Gregor in die Fußstapfen des „ersten Theologen“, des Evangelisten Ioannes, der von den Byzantinern $\pi \varrho \tilde{\omega} \tau о \varsigma$ $\theta \varepsilon \circ \lambda$ ó $\gamma \circ \varsigma$ und an einer Stelle, nämlich bei Georgios Bardanes im frühen 13. Jahrhundert, sogar mit dem Hapax $\pi \varrho \omega \tau о \theta \varepsilon о \lambda o ́ \gamma o \varsigma^{9}$ bezeichnet wird. In der Vita des Gregor von Nazianz aus der Feder des Gregorios Presbyter wird die Bezeichnung „Theologe“ besonders hervorgestrichen: Nur Gregor von Nazianz werde nach dem Evangelisten Ioannes „Theologe“ genannt. ${ }^{10}$ Später gesellt sich zu den beiden „Theologen“ Ioannes und Gregor auch ein „neuer“ Theologe hinzu, nämlich Symeon ó véos $\theta \varepsilon o \lambda o ́ \gamma o \varsigma$. Auch das Motiv des Donners setzt Ioannes und Gregor in Beziehung. Der Apostel bzw. Evangelist Ioannes ${ }^{11}$ wird (ebenso wie sein Bruder Jakob) seines Temperaments wegen im Neuen Testament von Jesus „Sohn des Donners“ (Mk 3,17) genannt; diese Bezeichnung lebt - wie

4 Pseudo-Nonniani in IV Orationes Gregorii Nazianzeni Commentarii ed. J. Nimmo Smith (CC SG 27), Turnhout 1992.

5 C. Macé, Les citations de Grégoire de Nazianze dans l'Edictum Rectae Fidei de Justinien, in: JÖB 52 (2002) 89-93.

6 Vgl. T. Špidlík, Per una rilettura di Gregorio Nazianzeno, in: Koเvwvía 5 (1981) 39-48, hier $45 f$.

7 ACO II 1,3,114,14.

8 Philostorgius, Kirchengeschichte. Mit dem Leben des Lucian von Antiochien und den Fragmenten eines arianischen Historiographen. Herausgegeben von J. Bidez; zweite Aufl. besorgt v. F. Winkelmann (Die griechisch christlichen Schriftsteller der ersten Jahrhunderte), Berlin 1972, VIII $11(112,1)$.

9 M. Roncaglia, Georges Bardanès, Métropolite de Corfou, et Barthelemy de l'ordre Franciscain. Les discussions sur le purgatoire, Rom 1953, 64,89.

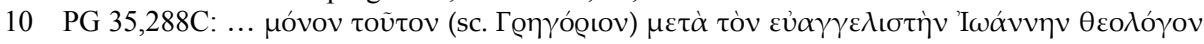
ỏvou $\alpha \sigma \theta \tilde{\eta} v \alpha$ เ.

11 Die beiden wurden traditionell sehr lange für ein und dieselbe Person gehalten. 
zahlreiche Beispiele zeigen - in der Rezeption der Byzantiner weiter. ${ }^{12}$ Gregor von Nazianz wird in der Nachfolge des Evangelisten Ioannes nicht nur zum „Theologen“, sondern erbt von diesem auch das Motiv des Donners. ${ }^{13}$ Theodoros

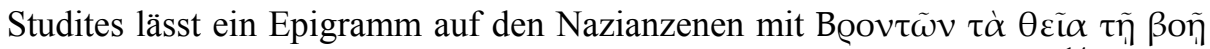

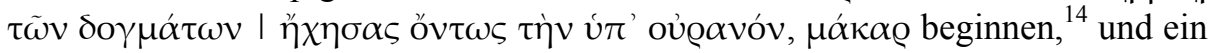
anonymes, noch unediertes Gedicht in einem Mailänder (Mediolan. Ambros. 1014, fol. II ${ }^{\mathrm{V}}$, [s. IX]) und Moskauer (Mosquen. Syn. 139, fol. 167 [s. VIII-IX])

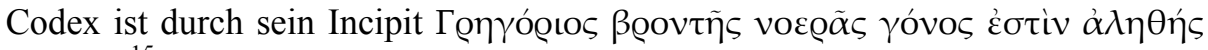
bekannt. ${ }^{15}$

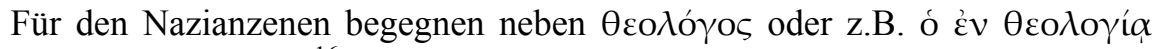

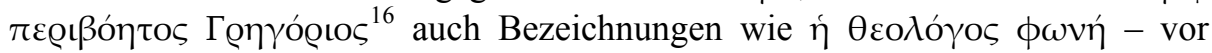

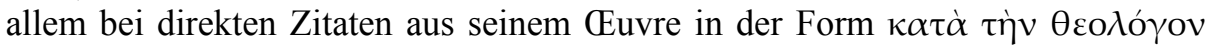

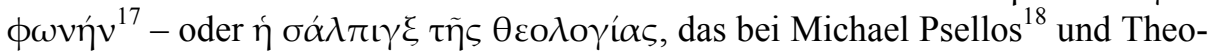
doros II. Laskaris belegt ist. Letztgenannte Stelle ist auch allgemein interessant: Laskaris schreibt an Georgios Mouzalon, dass er ihm ein Buch des Gregor von Nazianz geschickt habe. Er verlangt, dass dieser gestärkt durch die Lektüre des Theologen auf schnellstem Wege zu ihm kommen solle. ${ }^{19}$ In Parenthese sei erwähnt, dass auch bei Eustathios von Thessalonike ein Buch des Gregor von Nazianz erwähnt wird: Dieses sei laut Eustathios sehr prunkvoll gewesen, es kann jedoch in dem Kloster, in dem er es vermutet, nicht mehr gefunden werden. ${ }^{20}$

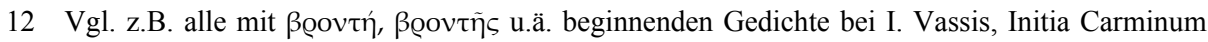
Byzantinorum (Supplementa Byzantina, Texte und Untersuchungen 8), Berlin/New York 2005, $110 \mathrm{f}$.

13 Dazu und zu den zwei im Folgenden genannten Beispielen M. Lauxtermann, Byzantine Poetry from Pisides to Geometres. Texts and Contexts (WBS XXIV/1), Wien 2003, I 170-173.

14 Ed. V. Somers, Quelques Poèmes en l'honneur de S. Grégoire de Nazianze: Édition critique, traduction et commentaire, in: Byz 69 (1999) 528-564, 539f. (Nr. 2) = P. Speck, Theodoros Studites. Jamben auf verschiedene Gegenstände. Einleitung, kritischer Text, Übersetzung und Kommentar (Supplementa Byzantina 1), Berlin 1968, 224 (Nr. LXVII). Speck schreibt in Vers 2 útov@avóv.

15 Ed. des Incipits bei Sajdak, Historia critica (s. Anm. 3), 270; vgl. auch Vassis, Initia (s. Anm. 12), 124.

16 Photius, Bibliothèque V, ed. R. Henry, Paris 1965, 228 (246b5). S. dazu den Beitrag von John Duffy in diesem Band, Proclus the Philosopher and A Weapon of Mass Destruction, 4.

17 Z.B. Nicephori Patriarchae Constantinopolitani refutatio et eversio definitionis synodalis anni 815, ed. J. M. Featherstone, Turnhout 1997, 13,90.

$18 \Psi \varepsilon \lambda \lambda$ ós. Michael Psellus de operatione daemonum cum notis Gaulmini curante J. F. Boissonade, Nürnberg 1838 (Reprint Amsterdam 1964), 125.

19 Theodori Ducae Lascaris Epistulae CCXVII, ed. N. Festa, Florenz 1898, 225 (ep. 172,12-15):

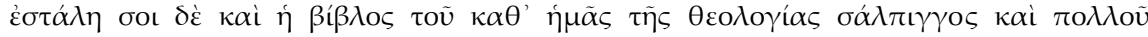

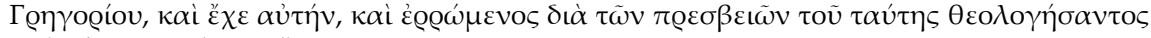

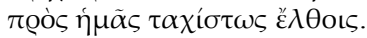

20 Eustathii Thessalonicensis de emendando vita monachica recensuit, germanice vertit, indicibus instruxit K. Metzler (CFHB XLV - Series Berolinensis), Berlin/New York 2006, §144 (160,10 
Gregor von Nazianz ist für die Byzantiner aber nicht nur der „Theologe“, sondern auch eine wichtige Instanz für ihr rhetorisches Schaffen. Der eigentliche antike Rhetor der Byzantiner ist Demosthenes, der bis ins 15. Jahrhundert rezipiert wurde. Zahlreich sind die Lobesworte, die über den Athener geschrieben wurden. Lukas Notaras im 15. Jahrhundert z.B. bewundert den Briefstil eines Freundes, da dessen Schreiben auch von Demosthenes oder Platon stammen könnte. ${ }^{21}$ Auch die spätantiken und byzantinischen Demosthenes-Biographien sind voll der Bewunderung für den antiken Rhetor. Einzig Ioannes Tzetzes stimmt nicht in den Chor der „Demosthenesverherrlichung“ ein, da seine Darstellung auf einer auf die Antike zurückgehenden Demosthenes-feindlichen Tradition beruht. ${ }^{22}$

Dem antiken heidnischen Demosthenes wird aber sehr bald ein christliches Gegenstück, nämlich Gregor von Nazianz, zur Seite gestellt. Mitunter fällt das Urteil sogar so aus, dass der Kirchenvater sein antikes Pendant übertrifft: Der im zehnten Jahrhundert tätige Rhetor Ioannes Sikeliotes ist zwar den antiken Instanzen wie Platon und Demosthenes gegenüber grundsätzlich wohlwollend eingestellt, zieht jedoch den „Theologen“ vor. Gregor übertrifft seiner Meinung nach nicht nur Platon ${ }^{23}$ in den Tugenden der Sprache und in der Theologie, sondern lässt auch Demosthenes als „bloßes Kind“ erscheinen. ${ }^{24}$ An anderer Stelle ist bei Sikeliotes Ähnliches zu lesen: Gregor übertrifft nicht nur Demosthenes, sondern auch alle anderen, und wenn man die Worte der beiden nebeneinander stellt, so erweist sich Demosthenes abermals als „bloßes Kind“. ${ }^{25}$ In dieselbe Richtung gehen auch die Urteile des Michael Psellos im 11. Jahrhundert: In einer kleinen

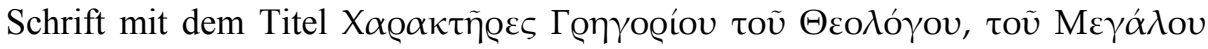

162,20); vgl. dazu M. Grünbart, Byzantium: a bibliophile society?, in: Basilissa 1 (2004) 113121, bes. $113 \mathrm{f}$.

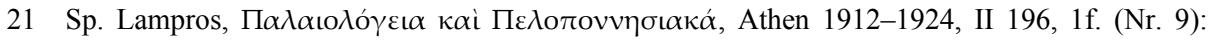

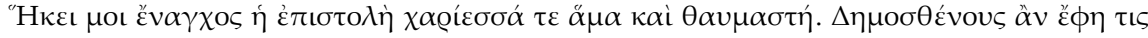

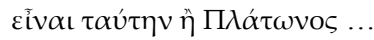

22 Vgl. E. Drerup, Demosthenes im Urteile des Altertums (von Theopomp bis Tzetzes: Geschichte, Roman, Legende) (Studien zur Geschichte und Kultur des Altertums XII, 1-2), Würzburg 1923 (Reprint Meisenheim 1968), 166-241.

23 Zum Vergleich mit Platon s.a. Michaelis Pselli theologica I, ed. P. Gautier, Leipzig 1989, 98,

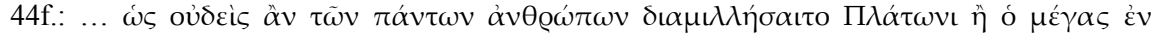

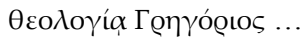

24 Chr. Walz, Rhetores Graeci ex codicibus Florentinis, Mediolanensibus, Monacensibus, Neapolitanis, Parisiensibus, Romanis, Venetis, Taurinensibus et Vindobonensibus, Tübingen 1832-36

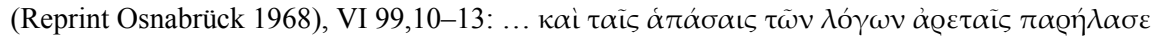

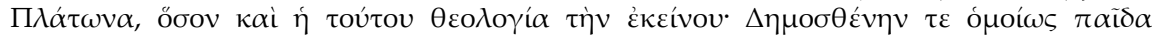
$\dot{\alpha} \pi \varepsilon ́ \delta \varepsilon เ \hat{\xi} \varepsilon$.

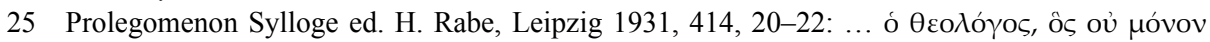

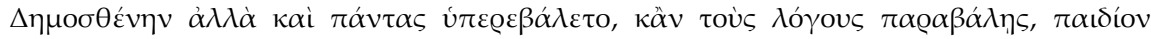

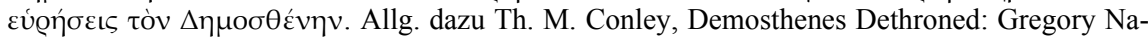

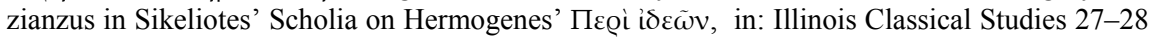
(2002-2003) 145-152. 


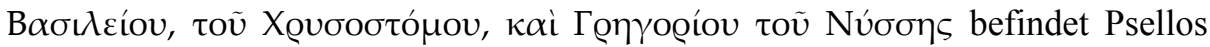
zunächst, dass neben dem Kanon antiker Musterautoren auch ein christlicher

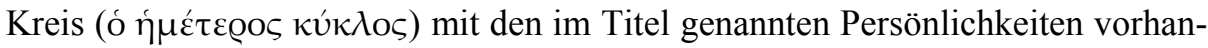
den ist. Gregor von Nazianz, der als erster mit den antiken Autoritäten in Relation gesetzt wird, wird zu dem, was Demosthenes für den paganen Bereich darstellt, nämlich zu einer Autorität in der Form eines „,christlichen Demosthenes“ ${ }^{26}$ Die Relation Demosthenes-Gregor ist bei Psellos keineswegs isoliert, sondern begegnet auch in seinem Gedicht über die Rhetorik: Als er bei der Beschreibung der Prokataskeue anlangt, meint er, dafür nicht ein Beispiel von Demosthenes anzuführen, sondern eines des „Theologen“. Dieser sei nämlich der allweise Philosoph (im Sinn von Theologe) und Rhetor. ${ }^{27}$ Danach zitiert Psellos ein Beispiel des Gregor von Nazianz für eine gelungene Prokataskeue. ${ }^{28}$

Ergänzend sei darauf hingewiesen, dass auch Libanios mit Demosthenes in Relation gesetzt wird. Im Abschnitt Пદ@i $\sigma v v \tau \alpha \dot{\xi} \xi \varepsilon \omega \varsigma^{29}$ der anonymen so genannten Lexica Segueriana (10. Jh. ?) wird Libanios an mehreren Stellen als „kleiner

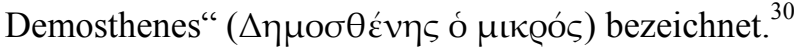

Neben Demosthenes und dem bereits erwähnten Platon kann es Gregor von Nazianz in der Wahrnehmung seiner späteren Rezipienten auch mit Homer aufnehmen. Aus der Feder des italienischen Philologen Scipio Carteromachus

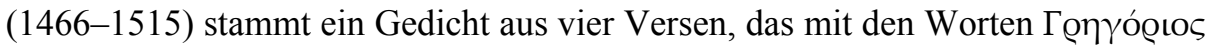

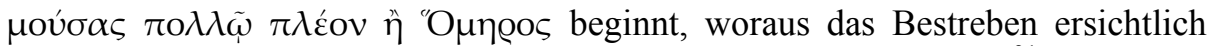
wird, Gregor von Nazianz auch über Homer triumphieren zu lassen. ${ }^{31}$ Eine ältere Version des Gedichtes mit demselben Incipit findet sich im Cod. Vat. Reg. Svec.

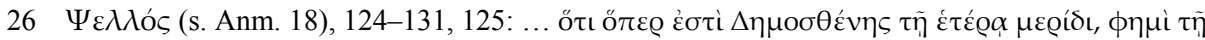

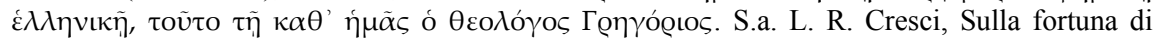
Gregorio di Nazianzo nel XII secolo, in: BollGrott n.s. 37 (1983) 3-17, hier 3f.

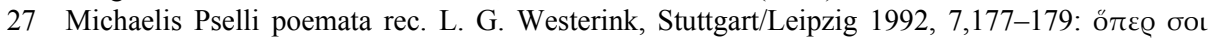

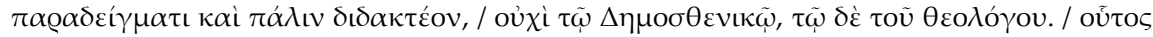

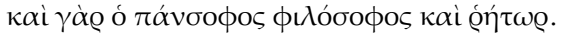

28 Psellos vergleicht auch in seiner Abhandlung über den rhetorischen Charakter des Nazianzenen Gregor mit antiken Autoritäten, darunter Demosthenes, s. Edition bei A. Mayer, Psellos' Rede über den rhetorischen Charakter des Gregorios von Nazianz, in: BZ 20 (1911) 27-100 (Text 4860, hier 48,1-26). Edition desselben Textes bei P. Levy, Michaelis Pselli de Gregorii Theologi charactere iudicium, Leipzig 1912 (Diss. Straßburg), 46-63.

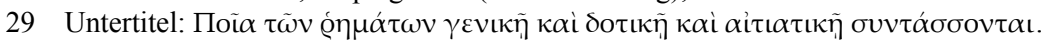

30 I. Bekker, Anecdota Graeca. Vol. I: Lexica Segueriana, Berlin 1814 (Reprint Graz 1965), 140,$13 ; 168,12 ; 172,7$.

31 Ed. in: PG 35,356C. 
166, f.13 ${ }^{\mathrm{v}}$ (I.) (s.XIV); ${ }^{32}$ auf dieses Incipit treffen wir auch im Cod. Ath. Laur. 1113, f.142 $2^{\mathrm{v}}$ (s.XVII). ${ }^{33}$

Welche Werke des Gregor von Nazianz wurden aber nun im Besonderen rezipiert? Dazu ist es notwendig, einen Blick auf die Zeugnisse zu werfen, die sich speziell auf das Euvre des Nazianzenen beziehen. Zunächst sei der Artikel

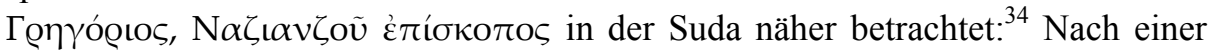
knappen Einleitung, in der Gregor als begabter Theologe und Rhetor apostrophiert wird, nennt der Suda-Artikel die besonders hervorzuhebenden Werke des Kappadokiers: An der Spitze stehen seine Gedichte und die Grabreden auf seinen Bruder Kaisarios, seinen Vater und seine Schwester Gorgonia. ${ }^{35}$ Es mag überraschen, dass bei dieser Auflistung Gregors Epitaphios auf seinen Freund Basileios den Großen nicht genannt wird. Überraschen deshalb, weil gerade diese Rede explizit in dem berühmten Traktat, der unter den Werken des Metropoliten von Korinth, Gregorios Pardos (11./12. Jh.), überliefert ist, erwähnt wird. In diesem Traktat, in dem u.a. die Vorbilder in den einzelnen Literaturgenera genannt werden, wird Gregor von Nazianz gleich mehrfach an prominenter Stelle erwähnt. Das Besondere an dieser Abhandlung liegt darin, dass nicht nur antike bzw. spätantike Autoren genannt werden, sondern auch echt „byzantinische“: Psellos wird als Vorbild bei Brief und Rede angeführt, Georgios Pisides, Nikolaos Kallikles und Ptochoprodromos bei der jambischen Dichtung. Als das herausragendste Werk des Gregor von Nazianz wird der Epitaphios auf seinen Freund Basileios den Großen genannt, der zusammen mit der Kranzrede des Demosthenes, dem Panathenaikos des Aristeides und der Grabrede des Psellos auf seine Mutter zum

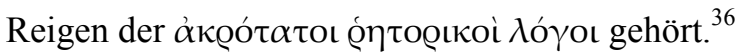

32 J. B. Pitra, Codices Manuscripti Graeci Reginae Svecorum et Pii PP. II Bibliothecae Vaticanae, Rom 1888, 113.

33 Spyridon [Lauriotes]/S. Eustratiades, Catalogue of the Greek Manuscripts in the Library of the Laura on Mount Athos (Harvard Theological Studies XII), Cambridge 1925 (Reprint New York 1969), 177; vgl. F. Halkin, Novum auctarium bibliothecae hagiographicae graecae, Brüssel 1984, 86 (730y).

34 Suidae Lexicon, Pars I, ed. A. Adler, Leipzig 1928 (Reprint Stuttgart 1989), 541f. ( $\gamma$ 450).

$35 \mathrm{Zu}$ den Grabreden Th. Hägg, Playing with expectations: Gregory's funeral orations on his brother, sister and father, in: Gregory of Nazianzus. Images and Reflections. Edited by J. Børtnes/Th. Hägg, Kopenhagen 2006, 133-151.

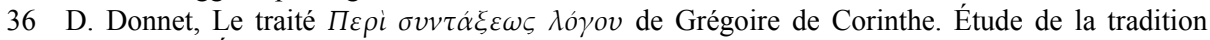

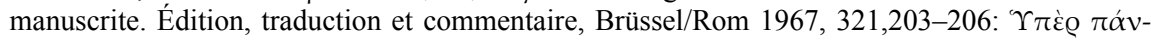

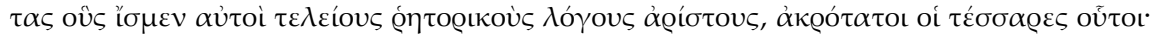

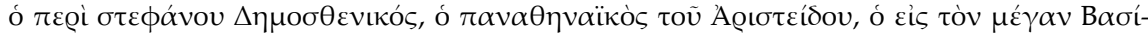

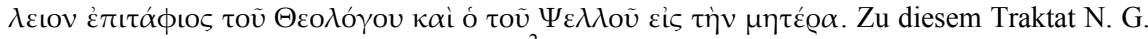
Wilson, Scholars of Byzantium, London ${ }^{2} 1996$, 184-190; zur Grabrede des Psellos nun J. Walker, These Things I Have Not Betrayed: Michael Psellos' Encomium of His Mother as a Defence of Rhetoric, in: Rhetorica 22/1 (2004) 49-101. 
Zahlreich sind in der Tat die Zitate, die den Epitaphien des Gregor von Nazianz entnommen sind. Untersuchungen haben ergeben, dass z.B. Psellos für die Grabrede auf seine Mutter Theodote reichlich den Epitaphios des Gregor von Nazianz auf seine Schwester Gorgonia herangezogen hat. ${ }^{37}$ Selbst im 17. Jahrhundert wird diese Rede noch als Beispiel herangezogen: Theophilos Korydaleus erwähnt in der an sein Briefhandbuch (1. Auflage London 1625) angefügten

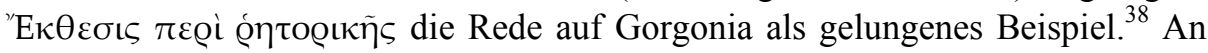
der Spitze der Rezeption steht jedoch Gregors Grabrede auf Basileios. Zitate davon begegnen nicht nur in so genannten ,rhetorischen“ Texten, sondern auch in der Historiographie. ${ }^{39}$ Eine wichtige Rolle spielt dabei die in dieser Rede und anderen Zeugnissen erwähnte „Freundschaft“ des Gregor mit Basileios, dem „Auge der Welt‘ ${ }^{\star 40}$, wobei diese, wie jüngst gezeigt wurde, in ihrer Intensität von Gregor erst nach dem Tod des Basileios besonders hervorgestrichen wurde. ${ }^{41}$ Die angeblich intensive Freundschaft zwischen den beiden nimmt der eifrige Gregorvon-Nazianz-Kenner Michael Psellos als Beispiel für seinen Briefadressaten Io-

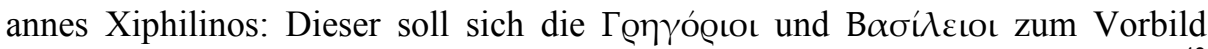
nehmen, die durch ihre Briefe ihre räumliche Entfernung aufgehoben hätten. ${ }^{42}$ Der Epitaphios auf Basileios wurde auch noch in postbyzantinischer Zeit genau studiert. Ein Beispiel: Eugenios Giannoules, Autor des späten 17. Jahrhunderts, bezeichnet Athen, das er durch einen längeren Aufenthalt sehr gut kennt, als „noch immer golden“ und als „gastfreundlich““ ${ }^{43}$ Die Bezeichnung ,gastfreundlich“ ( $\left.\pi \varrho \xi_{\varepsilon} \varepsilon v o \iota\right)$ für Athen ist nur in Gregors Epitaphios auf Basileios belegt, ${ }^{44}$ und „golden“ ( $џ \varrho v \sigma \alpha \tilde{)})$ ist eine Bezeichnung für Athen, die ebenfalls erstmals an

37 Vgl. Č. Milovanović, Псел и Григорије, нона и Теодота, in: ZRVI 23 (1984) 73-87 (mit engl. Zusammenfassung).

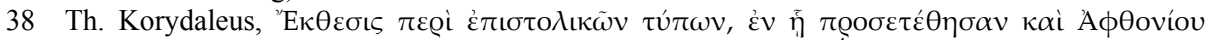

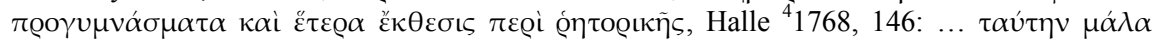

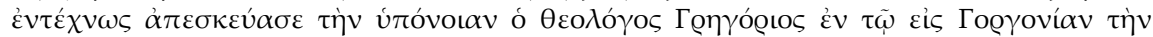

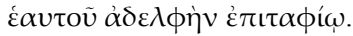

39 Z.B. Theophanes Continuatus, ed. I. Bekker, Bonn 1838, 63,22ff.

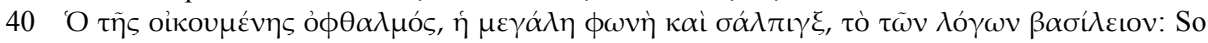
Gregor von Nazianz über Basileios in einem Brief an denselben, ed. P. Gallay, Gregor von Nazianz. Briefe (Die griechischen Schriftsteller der ersten Jahrhunderte 53), Berlin 1969, 42,8f. (Nr. XLVI).

41 N. McLynn, Gregory Nazianzen's Basil: The Literary Construction of a Christian Friendship, in: Studia Patristica 37 (2001) 178-193.

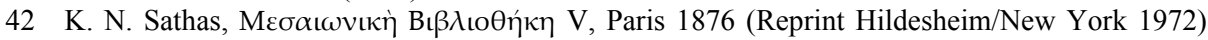

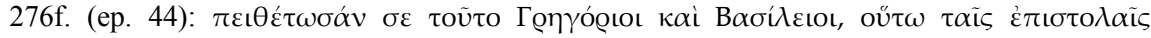

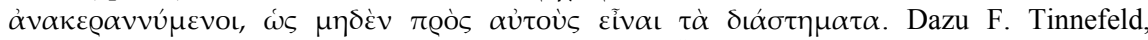
"Freundschaft" in den Briefen des Michael Psellos, in: JÖB 22 (1973) 151-168, hier 166f.

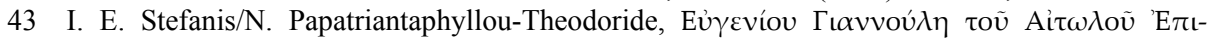

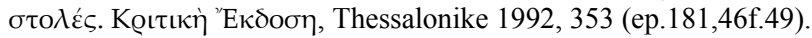

44 J. Bernardi, Grégoire de Nazianze. Discours 42-43 (SC 384), Paris 1992, 14,4-8 (p. 146ff.). 
dieser Stelle begegnet, ${ }^{45}$ und dann in Imitation von zahlreichen byzantinischen Autoren verwendet wird. ${ }^{46}$

À propos Briefe: Gregor von Nazianz ist auch in diesem Genre, wie wir bei Gregorios Pardos erfahren, ein beispielgebendes Vorbild. Der Kappadokier wird in der Epistolographie als erstes Archetypon neben Basileios dem Großen, seinem Bruder Gregor von Nyssa, Synesios, Libanios und Psellos genannt. ${ }^{47}$ Sein Briefœuvre umfasst 249 Schreiben; eine erste Sammlung dieser Briefe wurde bereits von Gregor selbst auf Bitten seines Großneffen Nikoboulos zusammengestellt. In einem Brief an diesen Nikoboulos unterbreitet Gregor auch wichtige

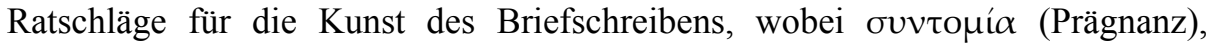

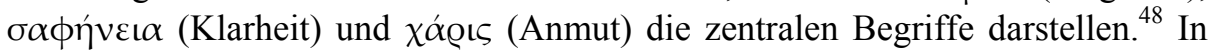
diesem Zusammenhang ist eine Stelle bei Arethas von Kaisareia interessant, der sich gegen den Vorwurf der $\alpha \sigma \alpha \dot{\phi} \phi \varepsilon \iota \alpha$ (mangelnde Klarheit) insofern wehrt, als seiner Meinung nach auch die Väter, speziell erwähnt er Gregor von Nazianz - im

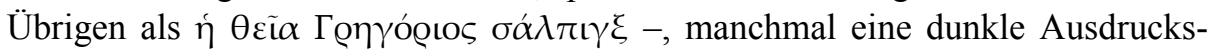
weise haben. ${ }^{49}$ Ein aussagekräftiger Hinweis für die Nachahmung der Briefe des Gregor von Nazianz ist ebenso wie bei anderen Autoren der Briefbeginn: Das

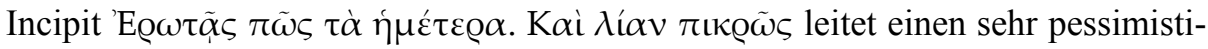
schen Brief Gregors ein, da er dort den Verlust von Basileios, Kaisarios und seinen Eltern heftigst bedauert. Daneben klagt er in diesem Schreiben auch über seine schlechte Gesundheit und sehnt sogar den Tod herbei, wenngleich er sich aber auch vor dem Jenseits fürchtet. ${ }^{50}$ Dieses Incipit begegnet in leicht veränderter Form bei Theophylaktos von Ohrid gegen Ende des 11. Jahrhunderts, der dem

45 Vgl. dazu A. Breitenbach, Das „wahrhaft goldene Athen“. Die Auseinandersetzungen griechischer Kirchenväter mit der Metropole heidnisch-antiker Kultur (Theophaneia. Beiträge zur Religions- und Kirchengeschichte des Altertums 37), Athen 2003, 150ff.

46 Die Beispiele reichen von den Theosophorum Graecorum Fragmenta (rec. H. Erbse, Stuttgart 1995, $\Delta$ 1-3) bis Ioannes Chortasmenos (ed. H. Hunger [WBS VII], Wien 1969, 202, 14f.). Dazu A. Rhoby, Spontane Anmerkungen zum ,goldenen“ Athen in Byzanz, in: JÖB 56 (2006) 53 57.

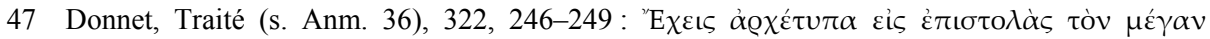

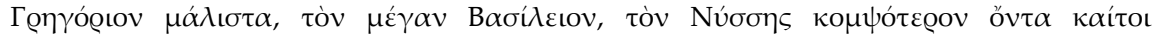

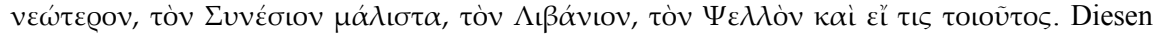
Text findet man auch bei Ioseph Rhakendytes, Walz, Rhetores graeci (s. Anm. 24), III 559,9-12 (XIV. Пвœì غ̇ंı

48 Gallay, Gregor von Nazianz (s. Anm. 40), 47f. (Nr. LI); s.a. G. T. Dennis, Gregory of Nazianzus and the Byzantine Letter, in: T. Halton/J. P. Williman (Hrsg.), Diakonia. Studies in Honor of Robert T. Meyer, Washington (D.C.) 1986, 3-13; A. Pliucas, The Theory of Style in the Letters of Gregory of Nazianzus, in: Annual of Medieval Studies at CEU 9 (2003) 9-27.

49 Arethae archiepiscopi Caesariensis scripta minora rec. L. G. Westerink, Leipzig 1968, I 186191, bes. 187,1ff.; vgl. dazu W. Hörandner, Rhetorik. Begriffsgeschichte: Byzanz, in: G. Ueding (Hrsg.), Rhetorik. Begriff - Geschichte - Internationalität, Tübingen 2005, 48-50, 49.

50 Gallay, Gregor von Nazianz (s. Anm. 40), 71 (Nr. LXXX). 


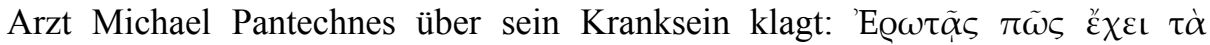

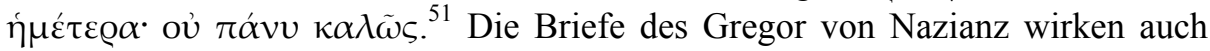
über das Ende von Byzanz hinaus. Sie gehören zusammen mit den Schreiben eines Synesios, Basileios, Ioannes Chrysostomos und Julian zu den hervorragenden Schreiben in der Zeit der Türkenherrschaft, wie die reiche handschriftliche Überlieferung beweist. ${ }^{52} \mathrm{Im}$ 17. und 18. Jahrhundert ist auch eine ausgedehnte Kommentatorentätigkeit zu den Briefen zu beobachten, außerdem werden neugriechische Paraphrasen angefertigt. ${ }^{53}$ Ein praktisches Beispiel für das Weiterleben der Briefe des Gregor von Nazianz über das Ende von Byzanz hinaus, wiederum identifiziert anhand des Initiums, begegnet in dem schon erwähnten Briefhandbuch des Theophilos Korydaleus, in dem sich auch einige Privatbriefe befinden. Wenn Korydaleus einen Brief an einen Freund mit den Worten 'E $\chi \varepsilon \iota \tau$

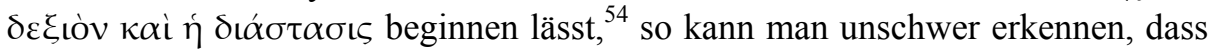
damit das Initium eines Briefes des Gregor von Nazianz nachgeahmt wird, das

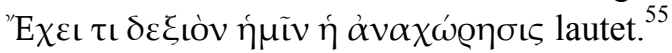

Die oben genannten Zeugnisse sind nur ein Ausschnitt aus dem schier unüberblickbaren Feld der Rezeption des Gregor von Nazianz, die auch mehr als eineinhalb Jahrtausende nach seinem Tod kein Ende gefunden hat.

51 P. Gautier, Théophylacte d'Achrida. Lettres. Introduction, texte, traduction et notes (CFHB XVI/2), Thessalonike 1986, 295 (Nr.48). Vgl. dazu M. Grünbart, Zum Verzeichnis der byzantinischen Briefanfänge (Epistularum byzantinarum initia), in: Göttinger Beiträge zur byzantinischen und neugriechischen Philologie 1 (2001) 47-49, $47 \mathrm{f}$.

52 Vgl. R. Romano, Gregorio Konstantas e la sua edizione delle epistole di Sinesio (Vienna 1792), in: XVI. Internationaler Byzantinistenkongress, Wien, 4.-9. Oktober 1981, Akten II/6 (= JÖB 32/6 [1982]), 239-248.

53 Lefherz, Studien (s. Anm. 3), 146f.

54 Vgl. M. Karpozilou, The epistolarion of Theophilos Korydaleus, in: Hell 49 (1999) 289-303, 300 (Incipit).

55 Gallay, Gregor von Nazianz (s. Anm. 40), 78 (Nr. XCIII). 
